\title{
Chronic obstructive pulmonary disease and heart failure: research and clinical practice in primary care
}

\author{
Francesco Chiumeo, ${ }^{1}$ Stefania Folloni ${ }^{2}$ \\ ${ }^{1}$ Regional Department of Primary Care and ${ }^{2}$ Regional Department of Continuing Heath Care, Italian Scientific Society for \\ Continuing Medical Education of General Practitioners (SNAMID), Trento, Italy
}

\begin{abstract}
The treatment of chronic obstructive pulmonary disease (COPD) and comorbidities, increasing with age, is the challenge that nowadays health care systems are facing to better care treat these patients. For this reason a clinical trial was conducted in the province of Trento by a group of 30 volunteer general practitioners members of SNAMID (Scientific Society for Continuing Medical Education of General Practitioners). The objectives were to identify: i) prevalence of COPD in patients (65-98 years) in the province of Trento; ii) presence and incidence of heart failure (HF) in COPD patients; iii) early detection of other chronic diseases; and iv) improving electronic medical records (EMR) as an innovation way of professional care management. From May 2011 to October 2013, 17 doctors completed the two-year work using the EMR. The studied patients were men and women (65-98 years), suffering from COPD; the considered data included: anthropometric information, smoking status, International Classification of Diseases (ICD)-9 diagnosis of COPD, HF and chronic diseases, specific blood and instrumental tests. The extracted results were then linked with data of sentinel therapies, collected by the EMR. The database obtained identified patients with COPD or HF not previously recognized with ICD-9 diagnosis. The study identified the sentinel drugs chosen for COPD and $\mathrm{HF}$, excluding other drugs not selective for the study or confusing for a proper statistical evaluation.
\end{abstract}

\section{Introduction}

Patients suffering from chronic obstructive pulmonary disease (COPD) frequently have comorbidities, which increase with age. The treatment of COPD and comorbidities is the challenge that nowadays the health care systems are facing and it is expected to persist also over the next years. ${ }^{1,2}$

Correspondence: Francesco Chiumeo, via Rosmini 12, 38122 Trento, Italy. Tel.: +39.0461.233310 - Fax: +39.0461.857045. E-mail: francesco.chiumeo@snamid.it

Stefania Folloni, via Roma 2, 38045 Civezzano (TN), Italy. Mobile: +39.348.5296692 - Fax: +39.0461.857046. E-mail: stefania.folloni@gmail.com

Key words: Chronic obstructive pulmonary disease; heart failure; comorbidities.

Conflict of interest: the authors declare no potential conflict of interest.

Received for publication: 20 November 2014.

Revision received: 9 January 2015.

Accepted for publication: 12 January 2015.

This work is licensed under a Creative Commons Attribution NonCommercial 3.0 License (CC BY-NC 3.0).

CCopyright F. Chiumeo and S. Folloni, 2015

Licensee PAGEPress, Italy

Italian Journal of Medicine 2015; 9:346-348

doi:10.4081/itjm.2015.560
According to the literature, the prevalence of heart failure (HF) in patients with COPD is between 18$25 \%$; therefore, it is necessary to recognize comorbid diseases in order to better treat these patients. ${ }^{2,3}$

The objectives of this observational study and clinical intervention were: i) the prevalence of COPD in patients, aged between 65 and 98 years in the province of Trento; ii) the presence and incidence of HF in COPD patients; iii) the early detection of other chronic diseases; iv) to improve electronic medical records (EMR) as a way of innovation in professional care management.

\section{Materials and Methods}

Thirty general practitioners (GPs) have voluntarily joined the research. All GPs work in the province of Trento, some of them are members of the Italian Scientific Society for Continuing Medical Education of General Practitioners (SNAMID), and others are only interested in the research at issue. The essential requirement was the use of an EMR, in order to extract directly research data with a specific query. The study lasted from May 2011 to October 2013 and just 17 doctors have completed the two-year work.

The studied patients, under the observation of these 17 practitioners, were men and women, aged between 65 and 98 years, suffering from COPD (Figure 1). The patient's selection was performed by using a specific query, which groups in anonymous database clinical 
and instrumental outcomes provided by the study protocol. The considered data were related to anthropometric information, smoking status, International Classification of Diseases (ICD)-9 diagnosis of COPD, heart failure and chronic diseases, frequent in the general population (diabetes mellitus, ischemic heart disease, hypertension, and osteoporosis). The data include also the results of specific blood and instrumental tests (NT pro-BNP, spirometry, echocardiography, ECG), in line with a diagnostic and therapeutic path, shared with the provincial hospitals. ${ }^{4-6}$

The extracted results regarding patients with COPD and heart failure have been linked up with the extracted data of sentinel therapies, with unique or selective indication for chronic obstructive pulmonary disease and heart failure. The drug treatment extracted from the medical record had to include at least three or more prescriptions in a year. The database built with this method has identified patients with COPD or heart failure (HF) not previously recognized with ICD-9 diagnosis. The sentinel drugs chosen for COPD were LABA, LABA + ICS, ICS co-administered with anticholinergics, for heart failure: $\beta$-blockers and cardiac glycosides. Other types of drugs for COPD and HF were excluded, because they were not sufficiently selective for the study and too confusing for a proper statistical evaluation. ${ }^{7}$

\section{Results}

The prevalence of COPD in 6511 patients over 65 years is $5.6 \%$ by extraction of ICD- 9 and $6.5 \%$ by extraction of sentinel therapies (Figure 1). There is a discrepancy between the prevalence of COPD/HF comorbidity measured with ICD-9, which stands at

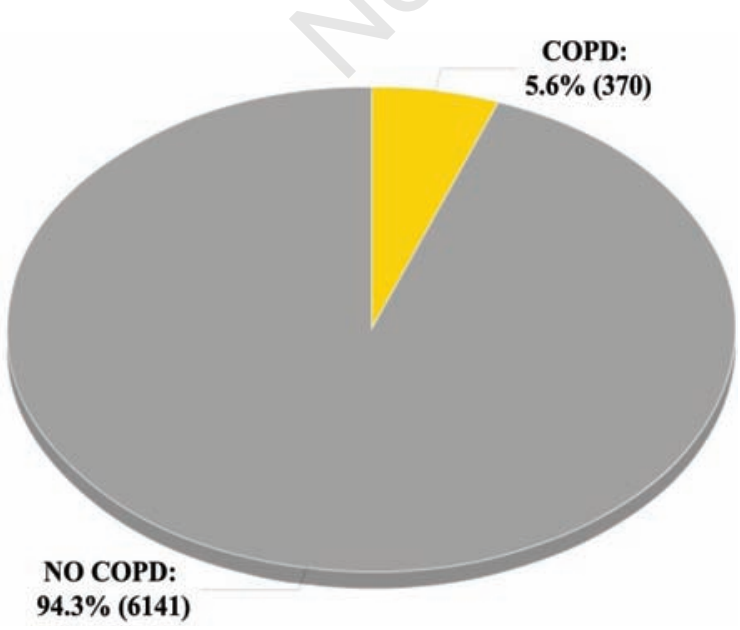

Figure 1. The prevalence of chronic obstructive pulmonary disease (COPD) in Trentino region, Italy (total no. of patients $=6511)$.
$11.0 \%$, and comorbidity measured by sentinel therapies, which stands at $24.8 \%$, according to the international literature. ${ }^{3,8}$ In our retrospective pragmatic study the patients were enrolled essentially by using the clinical and pharmacological criteria, because spirometry is not used adequately by generalist for the diagnosis of COPD.

Only $9.2 \%$ of COPD patients with ICD-9 diagnosis underwent spirometry with the Global Initiative for Chronic Obstructive Lung Disease (GOLD) gradation. The early detection of HF, if suspected, was performed by NT pro-BNP and, if the value was $>300 \mathrm{pg} / \mathrm{mL}$, by echocardiography according to HF guideline.

The database collected also allowed us to detect other comorbidities, particularly osteoporosis in an unexpected percentage (Figure 2).

The analysis of the therapy used by the GPs highlights that corticosteroids are the most widely used drugs, followed by anticholinergics and LABA-associated.

\section{Discussion}

The results are significant in the treatment of COPD patients with heart failure, as they lead to other indications regarding the diagnostic and therapeutic path, including the follow-up of early COPD and comorbidities.

The COPD-HF $24.8 \%$ result, in line with international literature data, was achieved by the evaluation of sentinel therapies, while the diagnostic data recorded according to ICD-9 are insufficient.

Research data show that COPD patients, detected by ICD-9 or sentinel therapies, are not so different. This means that this care model can improve the chronic pulmonary disease management by GPs.

It is important to underline that GPs do not usually use breath functional tests and miss to record anthropometric and smoking data.

This research highlights the insufficient use of electronic medical record for chronic diseases. This use can reduce the efficacy of the patient care.

\section{Conclusions}

The data collection and clinical evaluation with this methodology have made it possible to extract more information about the prevalence of important comorbidities, both in the general population and in patients with COPD. The comorbidity COPD-HF detected by the evaluation of a sentinel therapy in the province of Trento, is coherent with international data, but the diagnosis ICD-9 is inadequate. The use of spirometry for COPD and instrumental tests for HF is also insufficient. 


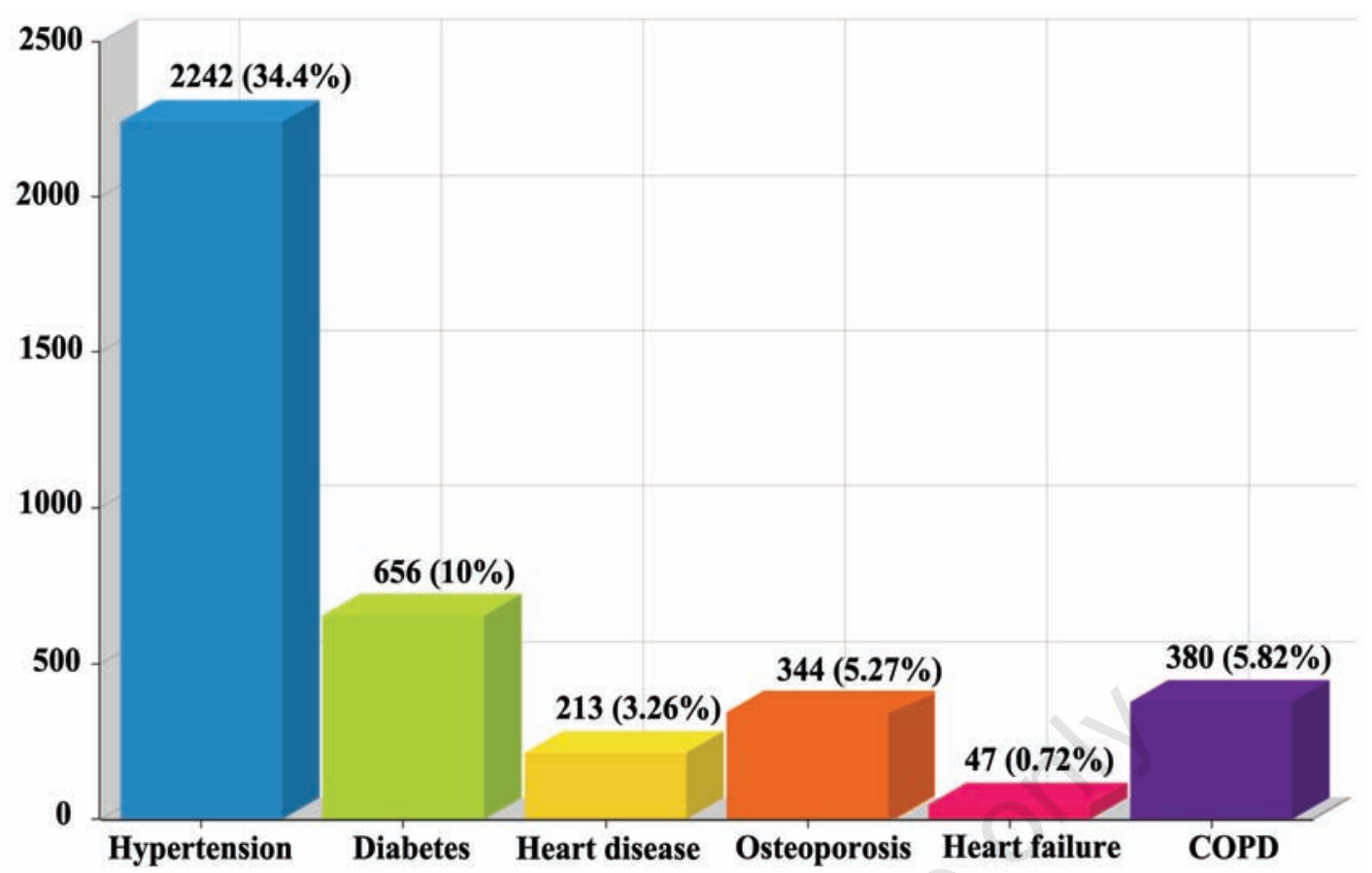

Figure 2. Colored bars show the absolute value and percentages of co-morbidity (total no. of patients=6519). COPD, chronic obstructive pulmonary disease.

Another significant finding in this study is the discrepancy in the chronic prescription of drugs used for the treatment of chronic bronchitis and heart failure, compared to the GOLD guideline. There is an unusual rate of osteoporosis comorbidity that is $84.47 \%$ of patients with chronic bronchitis.

The methodology proposed to detect the chronic bronchitis and heart failure comorbidity has been created to look over this comorbidity, looking for an application to other chronic diseases with systemic evolution, common in the elder population. Research in General Practice based on electronic medical records is a method of high significance. It allows you to see the real data, self-audit/audit that can provide a lot of information if properly coded. ${ }^{7,9,10}$

\section{References}

1. Istituto Nazionale di Statistica (ISTAT). Compendio statistico italiano. Roma: ISTAT; 2012. pp 50-52.

2. Rutten FH, Moons KG, Cramer MJ, et al. Recognising heart failure in elderly patients with stable chronic obstructicve pulmunary disease in primary care: cross sectional diagnostic study. BMJ 2005;331:1379.

3. Rutten, Cramer MJ, Grobbee DE, et al. Unrecognized heart failure in elderly patients with stable chronic obstruc- tive pulmonary disease. Eur Heart J 2005;26:1887-94.

4. McCullough PA, Hollander JE, Nowak RM, et al. uncovering heart failure in patients with a history of pulmonary disease: rationale for the early use of B-type natriuretic peptide in the emergency department. Acad Emerg Med 2003;10:198-204.

5. Rutten FH, Cramer MJ, Lammers JW, et al. Heart failure and chronic obstructive pulmonary disease: an ignored combination? Eur J Heart Fail 2006;8:706-11.

6. Van der Wel MC, Jansen RW, Bakx JC, et al. Non-cardiovascular comorbidity in elderly patients with heart failure outnumbers cardiovascular comorbidity Eur J Heart 2007;9:709-15.

7. Thierry H, Le Jemtel TH, Padeletti M, et al. Diagnostic and therapeutic challenges in patients with coexixtent chronic obstructive pulmunary disease and chronic heart failure. J Am Coll Cardiol 2007;49:171-80.

8. Siebeling van der Wal WM, Geskus RB, Zoller M, et al. Characteristics of Dutch and Swiss primary care COPD patients - baseline data of the ICE COLD ERIC study. Clin Epidemiol 2011;3:273-83.

9. Hawkins NM, Jhund PS, Simpson CR, et al. Primary care burden and treatment of patients with heart failure and chronic obstructive pulmonary disease in Scotland. Eur J Heart Failure 2010;12:17-24.

10. Barnett K, Mercer SW, Norbury M, Watt G. Epidemiology of multimorbidity and implications for health care, research, and medical education: a cross-sectional study Lancet 2012;7:380. 